\title{
Relations between pharmacokinetic parameters of carbamazepine and therapeutic response in bipolar patients
}

\author{
B.N. Saguem ${ }^{1}$, A. Braham¹, Z. Bouzaâbia1, C. Chbili², S. Saguem², S. Ben Nasr ${ }^{1}$. \\ ${ }^{1}$ Farhat Hached Hospital, Psychiatry Department, Sousse, Tunisia. \\ 2Faculty of medicine of Sousse, Metabolic Biophysics - Professional Toxicology and Applied Environmental Laboratory,
} Sousse, Tunisia.

\section{Background \& Objective}

- Carbamazepine (CBZ), an established therapeutic option in the treatment of bipolar disorder since the early 1970s [1,2], has become less and less prescribed in recent years, mainly because of the high frequency of relapses under this medication, and the difficulties of controlling its efficacy through standardized values of carbamazepine plasma levels.

- Efficient CBZ doses and efficient CBZ plasma levels were quite variable from one patient to an other, making it so difficult to optimize therapeutic response to $C B Z$ using $C B Z$ plasma levels only.

- This study aimed to address the relation between therapeutic response to $C B Z$ in bipolar patients and pharmacokinetic parameters of this medication including CBZ plasma levels, 10,11-epoxidecarbamazepine levels (CBZ-E) and the carbamazepine metabolic index.

\section{Methods}

- A prospective study was conducted. It included all euthymic bipolar patients who were exclusively treated with carbamazepine at a dosage allowing clinical stabilization over a minimum period of one month, due to carbamazepine self-induction properties.
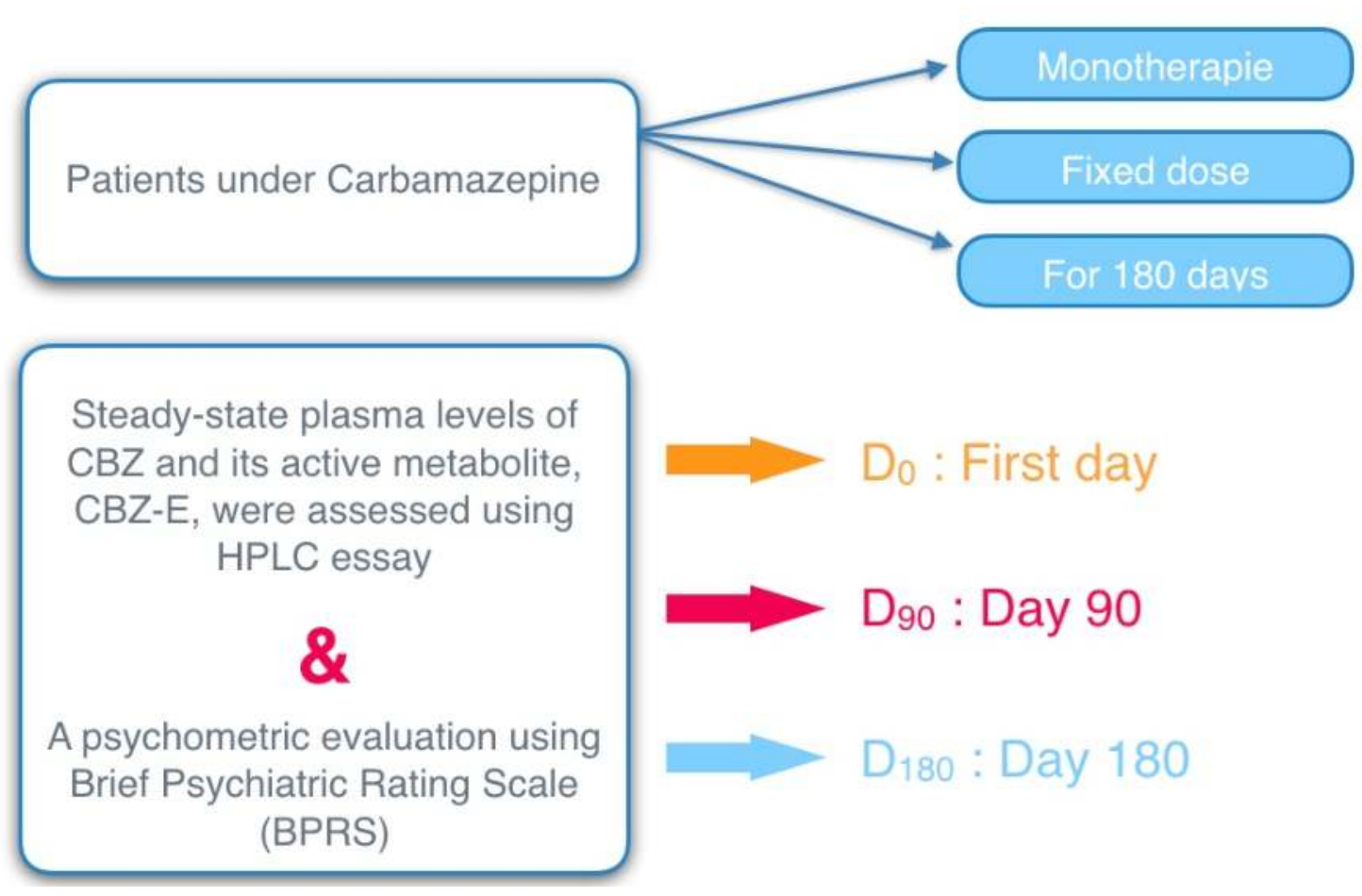

\section{Results \& Discussion}

- Thirteen patients, three women and 10 men, with bipolar I disorder were recruited, with a mean age of $43.85 \pm 10.73$ years.

- Table 1 illustrates CBZ plasma levels, CBZ-E plasma levels, the CBZ metabolic index and BPRS scores in the recruited bipolar I patients.

- Our study showed that optimum therapeutic response was observed among bipolar patients who had a plasma CBZ level of $7 \mu \mathrm{g} / \mathrm{ml}$ and a plasma metabolite level of $1.44 \mu \mathrm{g} / \mathrm{ml}$, simultaneously.

- Our findings are consistent with those showing no significant role of CBZ plasma levels in the prediction of therapeutic efficacy in bipolar patients [3,4]. Both CBZ and CBZ-E plasma levels should be fixed to achieve an optimum therapeutic response in bipolar I patients, taking into consideration the carbamazepine metabolic index.

- In order to reach the pharmacokinetic conditions required for optimum efficacy, inhibitor drugs of epoxide hydrolase, such as valproic acid [5], or inducer drugs of epoxide hydrolase, such as phenobarbital [6], might be co-administered with $\mathrm{CBZ}$ in order to adapt the plasma level of CBZ-E.
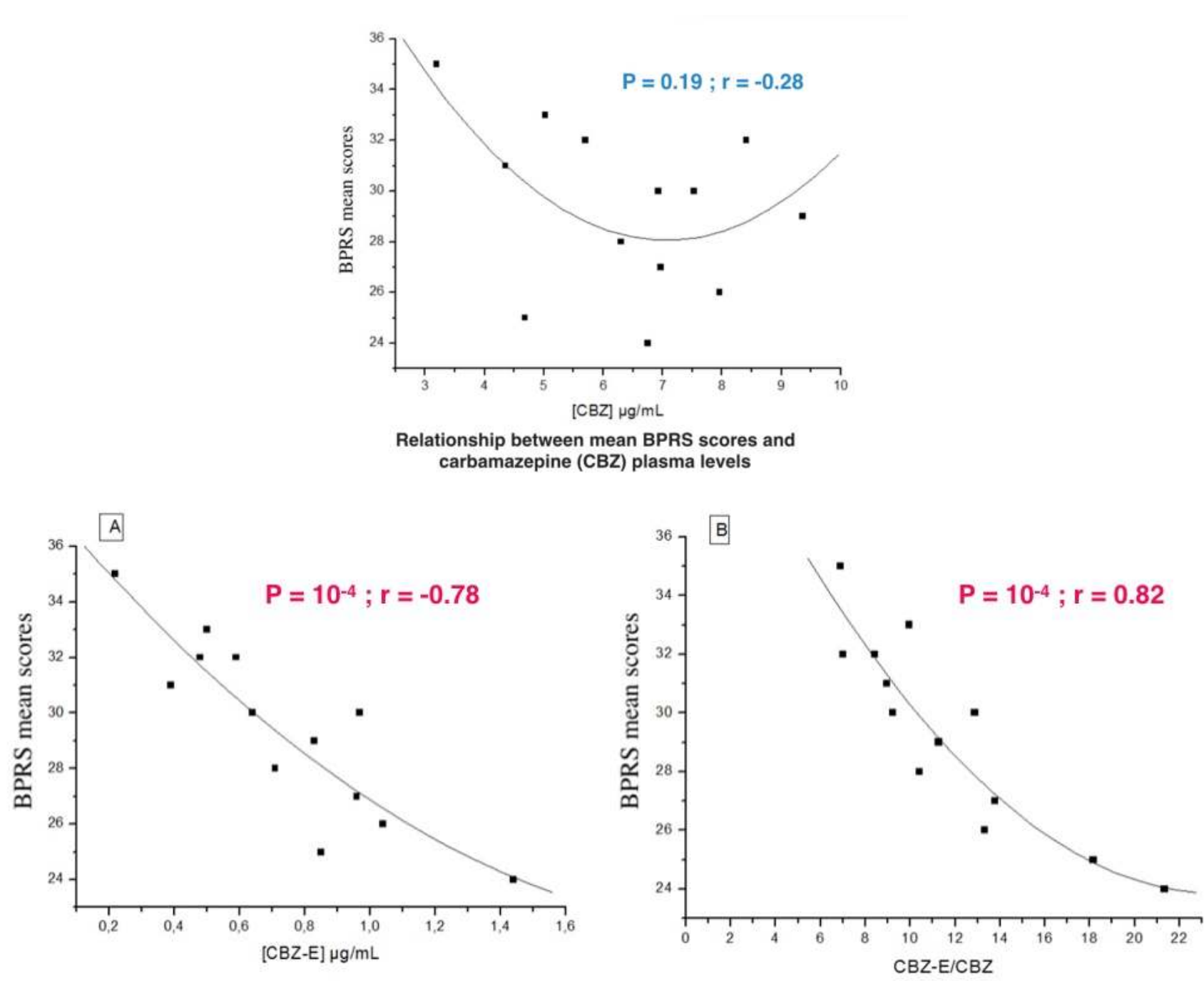

(A) Correlation between mean BPRS scores and mean plasma levels of 10,11epoxide-carbamazepine (CBZ-E);

(B) Correlation between mean BPRS scores and carbamazepine metabolic index

Table 1: CBZ plasma levels, CBZ-E plasma levels, the CBZ metabolic index and BPRS scores in the recruited bipolar I patients $(n=13)$

\begin{tabular}{ccccc} 
Patient & $\begin{array}{c}\text { Mean levels } \\
\text { of CBZp } \\
(\boldsymbol{\mu} \mathbf{g} / \mathrm{ml})\end{array}$ & $\begin{array}{c}\text { Mean levels } \\
\text { of CBZ-Ep } \\
(\boldsymbol{\mu g} / \mathrm{ml})\end{array}$ & $\begin{array}{c}\text { CBZ-E/CBZ } \\
(\%)\end{array}$ & $\begin{array}{c}\text { BPRS } \\
\text { mean scores }\end{array}$ \\
\hline 1 & 6.30 & 0.71 & 11.269 & 28 \\
\hline 2 & 8.41 & 0.59 & 7.015 & 32 \\
\hline 3 & 4.35 & 0.39 & 8.965 & 31 \\
\hline 4 & 5.70 & 0.48 & 8.421 & 32 \\
\hline 5 & 5.02 & 0.50 & 9.954 & 33 \\
\hline 6 & 3.19 & 0.22 & 6.896 & 35 \\
\hline 7 & 4.68 & 0.85 & 18.162 & 25 \\
\hline 8 & 6.75 & 1.44 & 21.333 & 24 \\
\hline 9 & 6.93 & 0.64 & 9.235 & 30 \\
\hline 10 & 7.80 & 1.04 & 13.333 & 26 \\
\hline 11 & 7.36 & 0.83 & 11.277 & 30 \\
\hline 12 & 7.53 & 0.97 & 12.881 & 30 \\
\hline 13 & 6.97 & 0.96 & 13.773 & 27 \\
\hline
\end{tabular}

CBZp: carbamazepine plasma levels; CBZ-Ep: plasma levels of 10,11-epoxidecarbamazepine; CBZ-E/CBZ: carbamazepine metabolic index; BPRS: Brief Psychiatric Rating Scale

\section{Proposed therapeutic protocol}

CBZ-E levels inferior than $1.4 \mu \mathrm{g} / \mathrm{ml}$

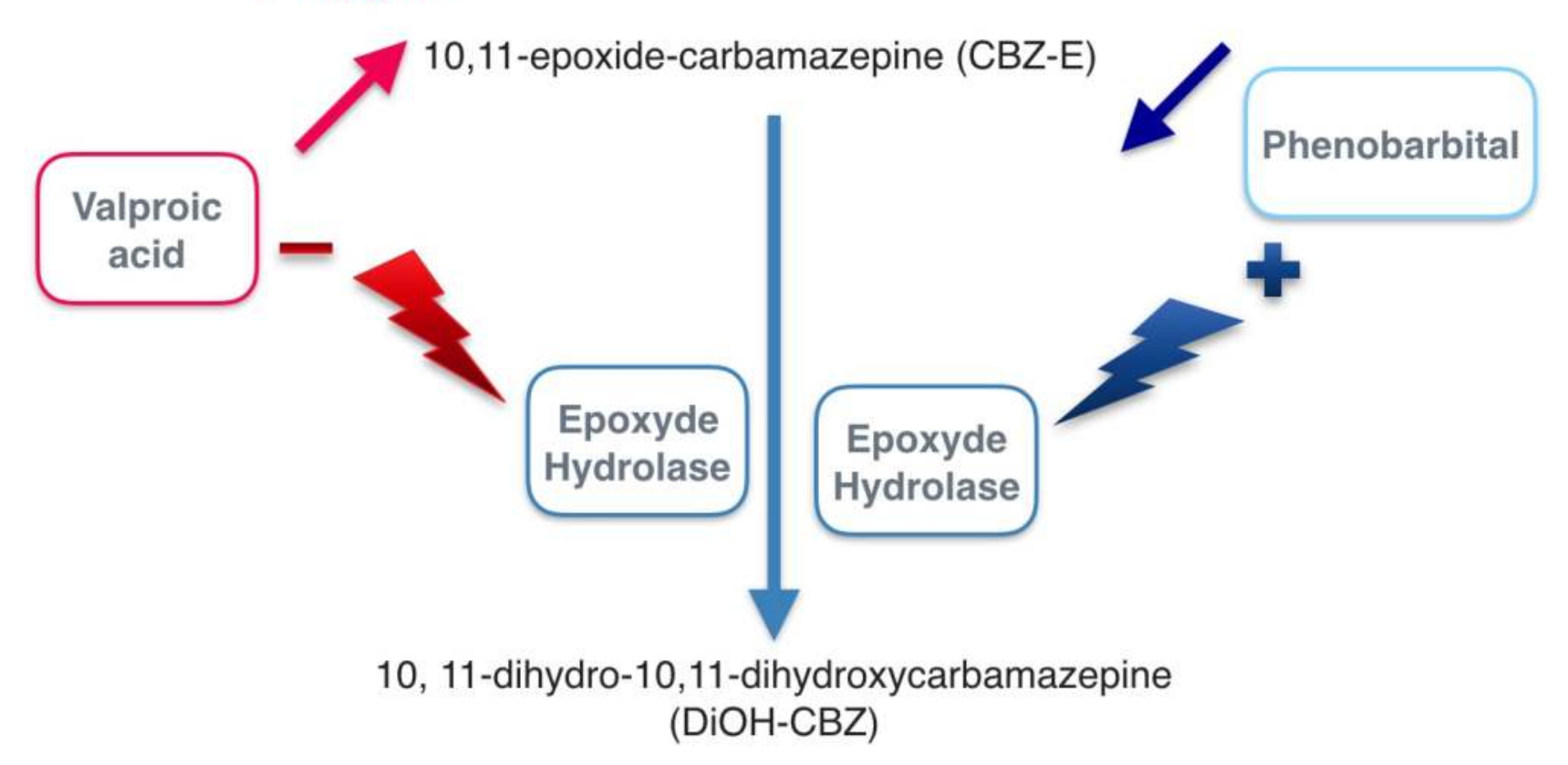

